\title{
Cirurgia ortognática para tratamento da síndrome de apneia obstrutiva do sono: relato de caso
}

\author{
Orthognathic surgery for obstructive sleep apnea syndrome \\ treatment: case report
}

\author{
Constanza Panissa* \\ Rafael Morawski* \\ Leonardo Tonietto ${ }^{* * *}$ \\ Vinicius Salim Silveira ${ }^{* * * *}$ \\ Jessica Lemos Gulinellit***** \\ Thiago Calcagnotto
}

\section{Resumo}

Objetivo: ilustrar, por meio de um caso clínico, os benefícios do tratamento da síndrome de apneia obstrutiva de sono (Saos) por intermédio da cirurgia ortognática bimaxilar, com o uso de biomateriais de reconstrução. Relato de caso: paciente encaminhado para avaliação de discrepância maxilomandibular em relação à base do crânio e para avaliação de queixa de ronco intenso durante a noite e intenso cansaço diurno. Após avaliação e exames complementares, o paciente foi diagnosticado com apneia obstrutiva do sono; assim, foi realizada a cirurgia de avanço bimaxilar como terapêutica resolutiva para o caso. Considerações finais: a Academia Americana de Medicina do Sono (AAMS) define Saos como uma doença que se caracteriza por episódios repetitivos de obstrução total (apneia) ou parcial (hipopneia) das vias aéreas superiores durante o sono. A cirurgia de avanço bimaxilar se apresenta como uma das melhores opções terapêuticas para os pacientes com essa síndrome. A cirurgia ortognática, incluindo seus diversos procedimentos, provoca importantes mudanças nas diferentes zonas das vias aéreas faríngeas. A cirurgia ortognática de avanço bimaxilar com mentoplastia de avanço foi eficaz, em longo prazo, no tratamento da Saos, e o uso simultâneo de biomateriais de substituição óssea aumenta a estabilidade óssea primária, acelera o processo de reparo ósseo e diminui a morbidade pós-operatória.

Palavras-chave: Apneia obstrutiva do sono. Avanço maxilar. Cirurgia ortognática.

\section{Introdução}

A síndrome da apneia obstrutiva do sono (Saos) é um problema de saúde pública não só pela sua elevada prevalência, mas também pela diversidade de danos crônicos e cumulativos que ocasiona na saúde geral do paciente. Essa síndrome se caracteriza por obstruções repetitivas das vias aéreas superiores em um determinado período de tempo ${ }^{1-3}$. O sintoma mais frequente é a sonolência diurna excessiva, além do ronco, que pode estar associado à Saos. A Academia Americana de Medicina do Sono define Saos como uma doença que se caracteriza por episódios repetitivos de obstrução total (apneia) ou parcial (hipopneia) das vias aéreas superiores durante o sono ${ }^{4}$. Epidemiologicamente, está associada à má qualidade de vida e pode levar a um maior risco de acidentes domésticos e veiculares, depressão, ansiedade, perda de memória e doenças cardiovasculares $^{5,6}$. A polissonografia é o padrão-ouro para avaliar a qualidade do sono do paciente e para diagnosticar a $\operatorname{Saos}^{2,7}$ e os demais distúrbios do sono.

Os tratamentos para esta doença envolvem desde alternativas conservadoras, como o uso de dispositivos intrabucais ${ }^{8}$, até procedimentos mais invasivos, como cirurgias ${ }^{9}$. Na Saos, a cirurgia ortognática tem como objetivo a redução da resistência da via aérea superior por meio da ampliação do

\footnotetext{
Aluna do curso de especialização em Cirurgia e Traumatologia Bucomaxilofacial (CTBMF), na Faculdade Tecnológica Dental (Fatec) do Centro de Excelência Educacional em Odontologia (Ceeo).

Cirurgião-dentista, graduado na Universidade Federal do Rio Grande do Sul.

Mestre em CTBMF, professor do curso de especialização em CTBMF, Fatec Dental Ceeo.

Doutor em CTBMF, professor do curso de especialização em CTBMF, Fatec Dental Ceeo.

Doutora em CTBMF, professora dos cursos de graduação em Odontologia e pós-graduação em CTBMF, Universidade do Sagrado Coração.

Mestre em CTBMF, coordenador do curso de especialização em CTBMF, Fatec Dental Ceeo.
} 
seu diâmetro, eliminando defeitos anatômicos que possam ocluir a luz aérea ${ }^{10}$. As osteotomias sagitais bilaterais para o avanço mandibular incrementam a dimensão anteroposterior da orofaringe e são alternativas para o tratamento da apneia obstrutiva do sono ${ }^{11}$.

A cirurgia do avanço bimaxilar consolida-se como uma das melhores opções terapêuticas para os pacientes com a Saos ${ }^{10,12}$. É uma opção cirúrgica eficaz para o tratamento da doença e que consegue a ampliação da via aérea superior por meio da expansão física do marco do esqueleto facial ${ }^{13}$, apresentando altas taxas de sucesso na melhora clínica do paciente ${ }^{14}$. Aliado a essa opção terapêutica, o uso dos cimentos cirúrgicos é uma opção viável e de imenso benefício para o paciente, pois, quando utilizados, evitam a formação de fibrose cicatricial além de guiarem a regeneração tecidual. Dentre os cimentos cirúrgicos, o cimento de fosfato de cálcio tem sido utilizado muito em cirurgias de ortopedia médica, sendo, em teoria, bem aplicado na odontologia na área de cirurgia e traumatologia bucomaxilofacial, para auxílio no reparo ósseo, principalmente facilitando a regeneração de um novo osso, bem como na estabilidade do segmento em que foi realizada a osteotomia ${ }^{15,16}$.

Este artigo tem como objetivo ilustrar, por meio de um caso clínico, a eficácia no tratamento da Saos com a cirurgia ortognática associada ao uso imediato de biomateriais de reconstrução óssea, como o cimento ósseo de fosfato de cálcio, a fim de melhorar a estabilidade óssea e a manutenção dos resultados em longo prazo. Para isso, a realização da polissonografia pré e pós-operatória é importante, visando a uma condição melhor de sono para o paciente e ao consequente tratamento para a Saos.

\section{Relato de caso}

Um paciente com 31 anos de idade, do sexo masculino, foi encaminhado para avaliação de discrepância maxilomandibular em relação à base do crânio e para avaliação de queixa de intensos ronco durante a noite e cansaço diurno. Ciente da pesquisa, o paciente assinou o termo de consentimento livre e esclarecido e foi informado do propósito e sentido da pesquisa realizada, concordando com os termos apresentados.

$\mathrm{Na}$ análise morfológica da face pré-operatória (avaliação frontal), vista na Figura 1A, o paciente apresentava simetria, com equilíbrio dos terços faciais, competência labial, presença do sulco nasogeniano com ligeira marcação e olheiras acentuadas. $\mathrm{Na}$ avaliação do perfil pré-operatório (Figura 1B), apresentava face convexa devido ao retro posicionamento bimaxilar, comprovada pela ausência da linha mento cervical. A análise dentária pré-operatória, em relação a molares, evidenciava classe I à direita (Figura 2A) e classe II à esquerda (Figura
2C). A avaliação de chave de caninos era classe II bilateral (Figuras 2A e 2C). A proporção entre arcadas era adequada transversalmente (Figura 2D).

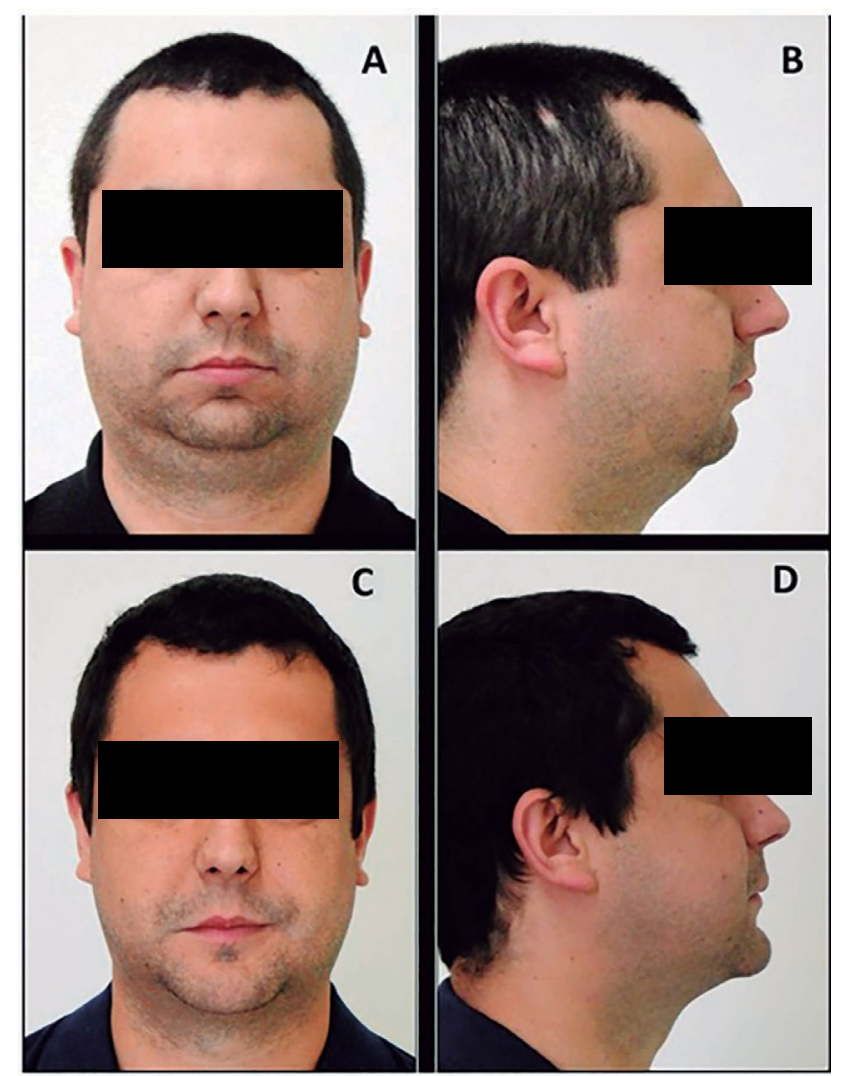

Figura 1 - A) Imagem clínica em perfil pré-operatório - B) Imagem clínica frontal pré-operatória - C) Imagem clínica em perfil pós-operatório - D) Imagem clínica frontal pós-operatória

Fonte: elaboração dos autores.

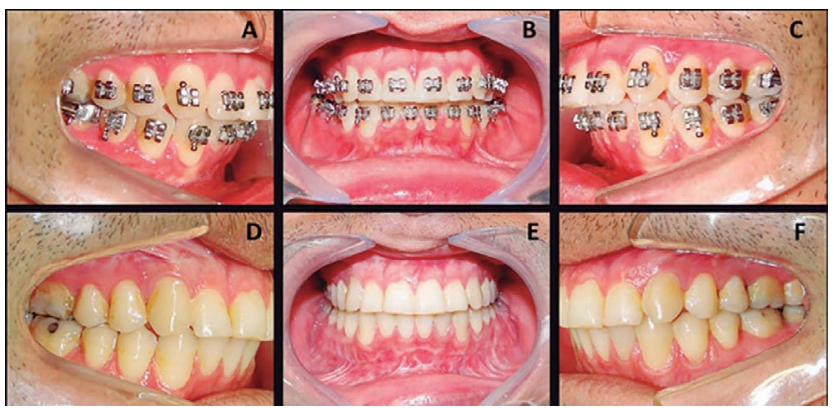

Figura 2 - A) Imagem clínica intrabucal do lado direito pré-operatória - B) Imagem clínica intrabucal anterior frontal pré-operatória - C) Imagem clínica intrabucal do lado esquerdo pré-operatória - D) Imagem clínica intrabucal do lado direito pós-operatória - E) Imagem clínica intrabucal anterior frontal pós-operatória - F) Imagem clínica intrabucal do lado esquerdo pós-operatória

Fonte: elaboração dos autores.

A telerradiografia de perfil pré-operatória ( $\mathrm{Fi}-$ gura 3A) evidencia retroposição da maxila e retroposição acentuada da mandíbula em relação à base do crânio. Tanto a maxila quanto a mandíbula se encontravam retraídas em relação ao complexo craniofacial, evidenciando estreitamento na região da hipofaringe. 


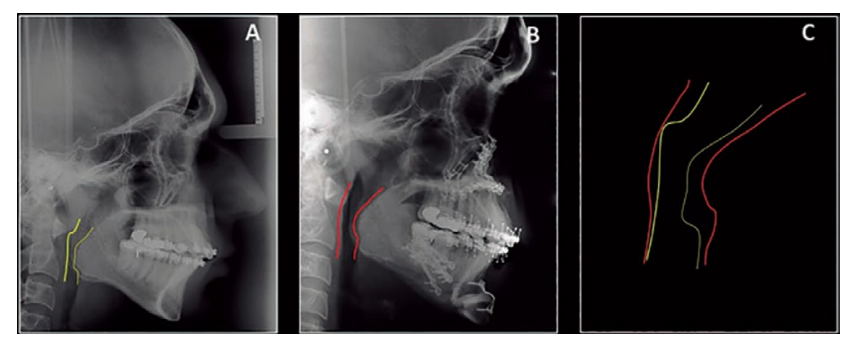

Figura 3 - A) Telerradiografia lateral pré-operatória com delimitação anteroposterior da via aérea (linhas amarelas) - B) Telerradiografia lateral pós-operatória com delimitação anteroposterior da via aérea (linhas vermelhas) - C) Sobreposição dos limites da via aérea pré-operatória (linhas amarelas) e limites da via aérea pós-operatória (linhas vermelhas)

Fonte: elaboração dos autores.

Para avaliação da qualidade do sono pré-operatória foi solicitada uma polissonografia ${ }^{2,7}$. Tal exame revelou que o paciente apresentava Saos com intensidade severa. Durante o período de exame (sete horas), o paciente não entrou no sono profundo e o índice de apneias e hipopneias foi de $512 \mathrm{em} 7$ horas de sono, das quais, 486 foram do tipo obstrutivas do sono de intensidade severa (Quadro 1).

A análise dos exames estabeleceu o diagnóstico de paciente classe II, birretruso e portador de Saos em grau severo. O tratamento proposto foi o ortocirúrgico, com realização de ortodontia pré-operatória, cirurgia ortognática para avanço bimaxilar e avanço de mento, buscando o aumento do espaço das vias aéreas e a finalização ortodôntica. $\mathrm{O}$ planejamento cefalométrico preditivo estabeleceu avanço maxilar de 7,0 $\mathrm{mm}$, avanço mandibular de $9,0 \mathrm{~mm}$ e avanço do mento de $6,0 \mathrm{~mm}$. Após a fixação dos segmentos ósseos maxilares e mandibulares nas posições estabelecidas no planejamento cirúrgico preditivo, as soluções de continuidade óssea foram recobertas com cimento ósseo de fosfato de cálcio (Osteovation Impact $^{\mathrm{TM}}$, Osteomed, Texas, USA) e membranas de colágeno (Bio Guide ${ }^{\mathrm{TM}}$, Geistlich Pharma, Wolhusen, Suíça). A região do gap do mento foi preenchida por bloco de fosfato de cálcio (Osteosynt ${ }^{\circledR}$, Einco Biomaterial LTDA., Belo Horizonte, Brasil) e recoberta com cimento ósseo de fosfato de cálcio (Osteovation Impact ${ }^{\mathrm{TM}}$, Osteomed, Texas, USA). O enxerto em bloco no gap do mento promoveu estabilidade e evitou que a tensão muscular gerada pelo grande avanço do mento o tracionasse para posterior. Ainda, o cimento ósseo, na mandíbula, promoveu estabilidade adicional das placas e dos parafusos de fixação e, na maxila, promoveu a reconstrução da parede da maxila e o adequado vedamento do seio maxilar (Figuras 4A e 4B).

A técnica utilizada diminuiu a ocorrência de recidiva da retroposição da maxila e da mandíbula e, mais importante, diminuiu o edema em face, que geralmente é causado pelo extravasamento de ar do interior do seio maxilar para os planos musculares, devido à osteotomia de maxila. $\mathrm{O}$ preenchimento com cimento ósseo de fosfato de cálcio ainda evita a formação de fibrose cicatricial ao invés de tecido ósseo novo.

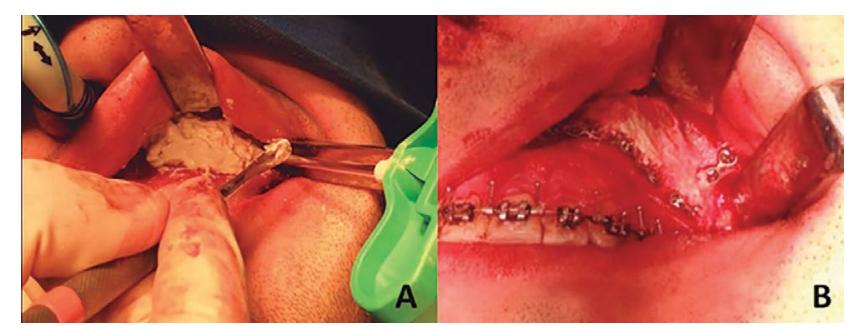

Figura 4 - A) Imagem clínica transoperatória evidenciando inserção do cimento ósseo de fosfato de cálcio na parede lateral de maxila sobre as placas - B) Imagem clínica transoperatória evidenciando cimento ósseo de fosfato de cálcio (Osteovation ImpactTM, Osteomed, Texas, USA) adaptado, após a cristalização, na parede anterior de maxila

Fonte: elaboração dos autores.

A avaliação dos exames pós-operatórios de imagem (exame de cefalometria, com análise de McNamara e polissonografia) evidenciou o aumento volumétrico da via aérea, conforme visto na telerradiografia pós-operatória de perfil (Figura 3B) e no desenho esquemático que compara os traçados dos limites anteroposterior da via aérea (Figura 3C).

O exame de polissonografia pós-operatória (Quadro 1), após dois anos, revelou que o paciente apresentou melhora significativa no quadro clínico, alcançando a cura da Saos, o que embasa e justifica o relato de remissão dos roncos durante o sono e a resolução das queixas de cansaço diurno. No exame, nota-se que houve uma melhora significativa na qualidade do sono, pois, durante as sete horas de exame, o paciente conseguiu entrar em sono profundo, e o índice de apneias e hipopneias foi de 90 . No exame pré-operatório, o paciente ficava $69 \%$ do seu sono em apneia; após a cirurgia, esse percentual decaiu para 6\% (Quadro 1). Toda essa melhora corrobora o relato do paciente, que relatou mais disposição diurna e um sono melhor durante a noite. Além da melhora do quadro grave de apneia, o paciente manteve a estabilidade oclusal.

Quadro 1 - Comparação dos parâmetros polissonográficos pré e pós-operatórios da cirurgia ortognática

\begin{tabular}{c|c}
\hline Pré-operatório & Pós-operatório \\
\hline 7 horas e 10 minutos & 7 horas e 2 minutos \\
00 horas e 00 minutos & 1 hora e 11 minutos \\
71 & 12 \\
73 & 11 \\
512 & 90 \\
$69 \%$ & $6 \%$ \\
$60 \%$ & $89 \%$ \\
515 & 117 \\
Intenso & Mínimo \\
\hline
\end{tabular}

Fonte: elaboração dos autores.

\section{Discussão}

A Saos é uma desordem caracterizada pela obstrução parcial ou total das vias aéreas pelos tecidos moles que constituem a faringe, gerando transtornos funcionais sistêmicos e cognitivos ${ }^{5,6}$. Apesar de 
não ser uma ação consciente, a alteração do padrão normal do sono prejudica o nível profundo do sono, de modo que o paciente apresenta constantemente a sensação de "noite mal dormida", evidenciando sintomas de fatiga e sonolência diurna permanente ${ }^{17}$.

A dimensão da faringe está diretamente relacionada ao posicionamento e à morfologia maxilomandibular e é altamente suscetível às alterações espaciais promovidas pela cirurgia ortognática ${ }^{13,17}$. No caso descrito, obteve-se aumento significativo do espaço faríngeo do paciente a partir do avanço bimaxilar associado ao avanço do mento, obtendo-se resultado positivo em relação ao tratamento da Saos em grau severo que o paciente apresentava. $\mathrm{O}$ aumento do espaço faríngeo refletiu melhora significativa no resultado pós-operatório da polissonografia, em que o número de paradas respiratórias passou de 512 para 90, respectivamente, nos períodos pré e pós-operatório. As apneias e as hipopineias pré-operatórias, que eram 73 , reduziram para 11 no período pós-cirurgia. Contudo, o resultado mais importante refere-se ao percentual de sono em que o paciente permanecia em apneia, que era, antes da cirurgia, de $69 \%$ e, com uma melhora significativamente importante, passou para $6 \%$.

$\mathrm{Na}$ análise cefalométrica de $\mathrm{McNamara}{ }^{18,19}$, os valores obtidos como padrão para o espaço faríngeo são $17,4 \mathrm{~mm}$ para superior e de $12 \mathrm{~mm}$ a $13 \mathrm{~mm}$ para inferior ${ }^{13,14}$. $\mathrm{Na}$ análise realizada na telerradiografia de perfil do paciente, houve evidência de incremento nos espaços faríngeos superior e inferior de $7 \mathrm{~mm}$ e de $8 \mathrm{~mm}$, respectivamente. Avaliando a cefalometria pós-operatória, observou-se um aumento de $10 \mathrm{~mm}$ no espaço aéreo superior e de $5 \mathrm{~mm}$ no espaço aéreo inferior ${ }^{20}$. Assim, esses valores comprovam a eficácia da cirurgia ortognática de avanço bimaxilar como forma de tratamento da Saos, o que é indicado por Capelloza ${ }^{17}$ para o tratamento de pacientes com perfil facial II birretruso.

$\mathrm{O}$ avanço maxilar e mandibular promove o alongamento do tecido mole do véu palatino, o que alonga também a musculatura palatoglosso e incrementa o suporte lingual, favorecendo uma maior abertura da via faríngea ${ }^{21}$, conforme foi realizado no caso ilustrado no artigo. A realização do avanço do mento, mais importante do que buscar a melhora estética do paciente, foi realizada tendo em vista a necessidade de obtenção de resultados mais estáveis em termos de abertura da via aérea, uma vez que o movimento de avanço da mandíbula e do mento influencia na posição do osso hioide, e no alongamento da musculatura supra-hioidea. Os métodos da mentoplastia adicionais aos procedimentos produzem variações maiores na via aérea faríngea e ajudam a evitar o colapso anteroposterior do espaço supraglótico $^{11}$.

O uso de substitutos ósseos, como o cimento de fosfato de cálcio (Osteovation Impact ${ }^{\mathrm{TM}}$, Osteomed, Texas, USA), usado como variação da técnica convencional em cirurgias ortognáticas, no caso relata- do, contribuiu para melhorar a estabilidade óssea e acelerar o processo de reparo ósseo. Os benefícios do uso de biomateriais são embasados pelas propriedades osteoindutoras e osteocondutoras desses materiais em diversos estudos ${ }^{22,23}$ e fornecem parâmetros biológicos para apresentação de seu uso como variação técnica em cirurgias que, como a ortognática, convencionalmente, não usam biomateriais. Outro aspecto importante em relação ao uso do cimento ósseo de fosfato de cálcio está relacionado ao vedamento da parede do seio maxilar, que é aberta devido à osteotomia da maxila e mantém uma passagem de ar para os planos de tecido mole. Essa passagem de ar, devido a fraturas cirurgicamente induzidas ou a traumas, pode acarretar e aumentar o edema em face ${ }^{24,25}$, que, em alguns casos, pode se exacerbar, levando o paciente a um pós-operatório mais crítico $^{26}$.

Assim, como visto no caso ilustrado neste artigo, o uso de cimento ósseo tanto em maxila quanto em mandíbula, além de aumentar a estabilidade, reduz o potencial de formação de edema em cirurgias ortognáticas. $\mathrm{O}$ paciente relatou sentir melhora significante na melhora da qualidade do sono e maior disposição diurna, visto que, como foi relatado anteriormente, ele procurou o serviço com queixas de ronco e cansaço diurno intensos. Dessa maneira, a indicação correta do procedimento aliada ao uso de biomateriais para auxiliar no reparo e pós-operatório são fundamentais para alcançar a excelência no tratamento do paciente.

\section{Considerações finais}

A cirurgia ortognática de avanço bimaxilar com mentoplastia de avanço é eficaz em longo prazo no tratamento da Saos, e o uso simultâneo de biomateriais de substituição óssea propõe uma variação de técnica cirúrgica que aumenta a estabilidade óssea primária, acelerando o processo de reparo ósseo e diminuindo a morbidade pós-operatória.

\section{Abstract}

Objective: the purpose of this study was to evaluate the benefits of treatment of Obstructive Sleep Apnea Syndrome (OSAS) through bimaxillary orthognathic surgery using reconstructive biomaterials. Case report: patient referred for evaluation of mandibular maxillary discrepancy in relation to the base of the skull and for evaluation of intense snoring complaint at night and intense daytime tiredness. After evaluation and complementary examinations the patient was diagnosed with OSAS, and thus, bimaxillary advancement surgery was performed as a therapeutic solution for the case. Final considerations: the American Academy of Sleep Medicine (AAMS) defines OSAS as a disease characterized by repetitive episodes of total obstruction (apnea) or partial (hypopnea) of the upper airways during sleep. Bimaxillary advancement surgery is presented as one of 
the best therapeutic options for patients with obstructive sleep apnea syndrome. Orthognathic surgery, including its various procedures, causes important changes in the different areas of the pharyngeal airways. The bimaxillary advancement orthognathic surgery with advancement was effective in the long term in the treatment of OSAS and the simultaneous use of biomaterials of bone replacement increase the primary bone stability, accelerate the bone repair process and decrease the postoperative morbidity.

Keywords: Obstructive sleep apnea. Maxillary advancement. Orthognathic surgery.

\section{Referências}

1. Campos CO, Soares YP, Colaço AXP, Cruz BMS. Síndrome da apneia obstrutiva do sono. Rev Inspirar Mov Saúde 2017; 12(1):29-36.

2. Balbani APS, Fomigoni GGS. Ronco e síndrome da apnéia obstrutiva do sono. Rev Assoc Med Brasileira 1999; 45(3):273-8.

3. Martins AB, Tufik S, Moura SMGPT. Síndrome da apnéia-hipopnéia obstrutiva do sono. Fisiopatologia. J Bras Pneumol 2007; 33(1):93-100.

4. Carrillo JLAF, Reyes M, Castorena A, Vázquez JC, Torre-bouscoulet L. Síndrome de apnea obstructiva del sueño en población adulta. Neumol Cir Torax 2010; 69(2):103-15.

5. Chang CP, Li TC, Hang LW, Liang SJ, Lin JJ, Chou CY, et al. The relationships of sleep apnea, hypertension, and resistant hypertension on chronic kidney disease. Medicine (Baltimore) 2016; 95(23):e3859 1-5.

6. Uyar M, DAVutoglu V. An update on cardiovascular effects of obstructive sleep apnoea syndrome. Postgrad Med J 2016; 92(1091):540-4.

7. Togeiro SMGP, Smith AK. Diagnostics methods for sleep disorders. Rev Bras Psiquiatr 2005; 27(1):8-15.

8. Whitla L, Lennon P. Non-surgical management of obstructive sleep apnoea: a review. Paediatr Int Child Health 2016; 37(1)1-5.

9. Gerbino G, Gervasio FC, Blythe J, Bianchi FA. The Management of Iatrogenic Obstructive Sleep Apnea Syndrome Following Bimaxillary Surgery in a Patient with Cleft Lip and Palate. J Craniofac Surg 2016; 27(5):1286-8.

10. Almuzian M, Almukhtar A, Ju X, Al-hiyali A, Benington P, Ayoub A. Effects of Le Fort I Osteotomy on the Nasopharyngeal Airway-6-Month Follow-Up. J Oral Maxillofac Surg 2016; 74(2):380-91.

11. Rodríguez ARV, Moreno RF, Lagos OAV. Cambios en la dimensión de la vía aérea faríngea basado en estudio cefalométrico, posterior a cirugía ortognática. Rev Colombiana Invest Odontología 2012; 3(8):1-10.

12. Islam S, Taylor CJ, Ormiston IW. Effects of maxillomandibular advancement on systemic blood pressure in patients with obstructive sleep apnoea. Br J Oral Maxillofac Surg 2015; 53(1):34-8

13. Zaghi S, Holty JE, Certal V, Abdullatif J, Guilleminault C, Powell NB, et al. Maxillomandibular advancement for treatment of obstructive sleep apnea: a meta-analysis. JAMA Otolaryngol Head Neck Surg 2016; 142(1):58-66.

14. Won CH, Li KK, Guilleminault C. Surgical treatment of obstructive sleep apnea: upper airway and maxillomandibular surgery. Proc Am Thorac Soc 2008; 5(2):193-9.
15. Da Costa G, Pare A, Goga D, Sury F, Laure B. CT-scan evaluation of calvarial bone donor site reconstruction using calcium phosphate cement. Rev Stomatol Chir Maxillofac Chir Orale 2014; 115(3):140-4

16. Stetzer K, Cooper G, Gassner R, Kapucu R, Mundell R, Mooney MP. Effects of fixation type and guide tissue regeneration on maxillary osteotomy healing in rabbits. J Oral Maxillofac Surg 2002; 60(4):436-7.

17. Capelozza LDAA, Pinto F, Nary H, Ávila L, De Almeida M. Tratamento ortodôntico cirúrgico da birretrusão associado à Saos: relato de caso. Orthodontic Science and Practice 2012; 19(5):311-24

18. Bolender Y, Deblock L, Theuveny T, Bolender CJ, Turlot JC Comparison of the cephalometric analyses of Bjork, Ricketts and Sassouni with the measurements of McNamara and Wits. Rev Orthop Dento Faciale 1986; 20(2):251-71.

19. McNamara JA JR. A method of cephalometric evaluation. Am J Orthod 1984; 86(6):449-69.

20. McNamara JA JR. Influence of respiratory pattern on craniofacial growth. Angle Orthod 1981; 51(4):269-300.

21. Iglesia FLA. Apnea obstructiva del sueño y ortodoncia. Rev Esp Ortod 2010; 40(2):129-32.

22. Barradas AM, Yuan H, Van Blitterswijk CA, Habibovic P. Osteoinductive biomaterials: current knowledge of properties, experimental models and biological mechanisms. Eur Cell Mater 2011; 21:407-29.

23. Habibovic P, DE Groot K. Osteoinductive biomaterials - properties and relevance in bone repair. J Tissue Eng Regen Med 2007; 1(1):25-32.

24. Kurunadalingam H, Beegun I, Dubrey SW. Facial emphysema following nasal fracture. Br J Hosp Med (Lond) 2015; 76(2):113

25. Lim JL. Periorbital edema after dental extraction: a case study. Aust Fam Physician 2014; 43(8):543-4

26. Puricelli E, Ponzoni D, Artuzi FE, Martins GL, Calcagnotto T. Clinical management of angioneurotic oedema patient post-orthognathic surgery. Int J Oral Maxillofac Surg 2011; 40(1):106-9.

\section{Endereço para correspondência:}

\section{Thiago Calcagnotto}

Rua São João, 942

CEP: 93010-250, São Leopoldo, RS, Brasil

Telefone: (51) 991747104

E-mail: tcalcagnotto@hotmail.com

Recebido: 01/11/17. Aceito: 15/12/17. 